\title{
Benchmark calculation for radioactivity inventory using MAXS library based on JENDL-4.0 and JEFF-3.0/A for decommissioning BWR plants
}

\author{
Ken-ichi Tanaka \\ The Institute of Applied Energy, 1-14-2 Nishi-shinbashi, minato-ku, Tokyo, 105-003, Japan \\ Tokyo Institute of Technology, 2-12-N1-9 Ookayama, Meguro-ku, Tokyo 152-8550, Japan
}

\begin{abstract}
We performed benchmark calculation for radioactivity activated in a Primary Containment Vessel (PCV) of a Boiling Water Reactor (BWR) by using MAXS library, which was developed by collapsing with neutron energy spectra in the PCV of the BWR. Radioactivities due to neutron irradiation were measured by using activation foil detector of Gold $(\mathrm{Au})$ and Nickel (Ni) at thirty locations in the PCV. We performed activation calculations of the foils with SCALE5.1/ORIGEN-S code with irradiation conditions of each foil location as the benchmark calculation. We compared calculations and measurements to estimate an effectiveness of MAXS library.
\end{abstract}

\section{Introduction}

According to the Japanese law, any operating organization of decommissioning of nuclear facilities such as electric power companies needs to submit an application for decommissioning and to receive the authorization. Tasks for the decommissioning planning start with radiological characterization of the facilities. Reliable information about radioactivity inventory obtained from the task is much important to establish a decommissioning strategy and to perform decommissioning activities safely and rationally.

We performed benchmark calculation of radioactivity of activation foils irradiated by neutrons in a Primary Containment Vessel (PCV) of a Boiling Water Reactor (BWR) by using SCALE5.1/ORIGEN-S code [1] with MAXS library [2]. MAXS library is activation cross section data library for activation calculation in the PCV of the BWR.

Neutron energy spectra in the PCV of the BWR tend to show a peak in epi-thermal energy range. Thus, use of ORIGEN-S cross section library that was developed by collapsing cross sections with a typical neutron energy spectrum of a Light Water Reactor (LWR) is considered to be inappropriate under the condition in the PCV. In particular, applying it to estimations of a radionuclide generated by a reaction that has threshold energy would make a large ambiguity. To give values of cross sections by weighting with the neutron energy spectra in the PCV would be one of solutions.

A new multi-group neutron activation cross section library MAXS [2] was developed to solve the 
problem mentioned above in JAEA based on JENDL-4.0 and JEFF-3.0/A. Activation cross sections are collapsed by using various neutron energy spectra in the PCV (e.g. core internals, a reactor pressure vessel, PCV internals and a Biological Shielding Wall (BSW)). Furthermore, data of cross section for a number of reactions such as $(n, \gamma),(n, p),(n, \alpha),(n, 2 n)$ and so on were considered to generate the library.

We performed activation calculations at activation foils in the PCV of the BWR by using SCALE5.1/ORIGEN-S with MAXS library as a benchmark calculation. We compared calculated radioactivities with measured ones to estimate the effectiveness.

\section{Outline of benchmark problem}

\subsection{A typical spectrum of a LWR and neutron energy spectra in the PCV of the BWR}

A typical spectrum of the LWR used as a weighting function in a generation of ORIGEN library is shown in Figure 1 [3].

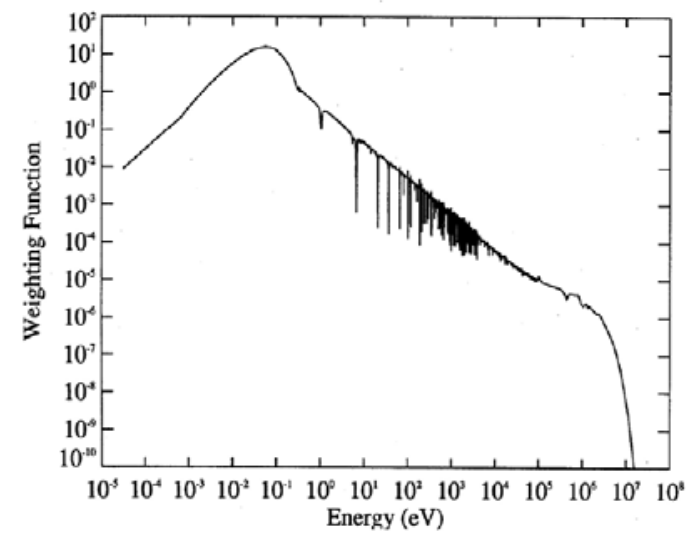

Figure 1 A typical neutron energy spectrum of a Light Water Reactor used as a weighting function in a generation of ORIGEN library [3]

The typical spectrum is comprised of three kinds of spectra; thermal Maxwellian spectrum in thermal energy range, 1/E spectrum in epi-thermal energy range and fission spectrum in fast energy range. The shape of the typical spectrum has been given as results from neutron transport phenomena which are supposed to be observed in the LWR. Emitting neutrons by nuclear fission and moderation by light water are being considered in the phenomena. However, the phenomena would be too simple to apply to a complicated structure like inside the PCV of the BWR.

Neutron energy spectra in the PCV of the BWR tend to show a peak in epi-thermal energy range as seen in Figure 2 [4]. The right side of Figure 2 shows position of neutron energy spectra drawn in the left side. Positions (1), (2), (3) and (4) are in a Reactor Pressure Vessel (RPV). Fission spectrum is dominant in the spectra at these positions. The spectra show a peak in epi-thermal energy range at positions (5) and (7) which are located inside the PCV. The last one at position (6) is in concrete of a Biological Shielding Wall (BSW). A shape of the spectrum in the BSW is almost similar to that of the typical spectrum. 
Various kinds of neutron transport phenomena occur in the PCV. Spectra around a reactor core such as positions (1), (2), (3) and (4) are almost similar to a fission spectrum. Neutrons leak from the reactor core and are moderated in coolant water flowing through an area between a shroud, which surrounds the reactor core, and a reactor pressure vessel (RPV). Although a spectrum in the area shows a shape which similar to the typical LWR spectrum, thermal neutrons are absorbed during penetrating the RPV. Because of a mechanism of the transport phenomena mentioned here, spectra in the PCV tend to show a peak in epi-thermal range such as positions (5) and (7).

According to a comparison between the shape of the typical spectrum of a LWR and those of spectra in the PCV of the BWR, it would be inappropriate to apply ORIGEN library, which is generated with the typical spectrum, to activation calculation in the PCV. In the benchmark calculations, we applied MAXS library, which is generated by collapsing with neutron energy spectrum at position (7), to activation calculation for radioactiveities measured with activation foils in the PCV.
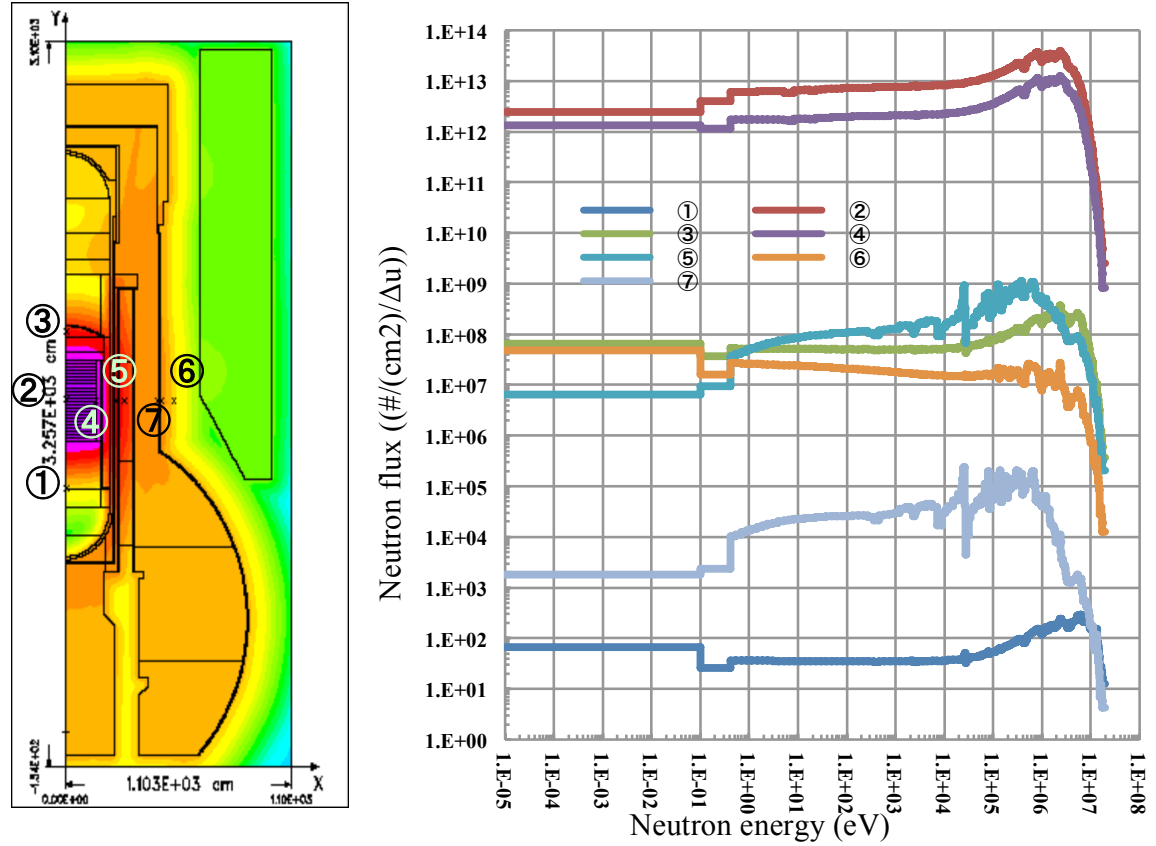

Figure 2 Neutron energy spectra in the PCV of the BWR

\subsection{Radioactivity measurements with activation foil in the PCV}

We measured radioactivities induced by neutrons in the PCV with three types of activation foils as foil activation detectors; Gold $(\mathrm{Au})$ foils, Gold foils covered by Cadmium $(\mathrm{Au}+\mathrm{Cd})$ foils and Nickel (Ni) foils. We can obtain radioactivities induced by neutron-flux of thermal energy range (thermal-flux: energy less than $1.0 \mathrm{eV}$ ) from a difference between measurements of $\mathrm{Au}$ foils and $\mathrm{Au}+\mathrm{Cd}$ foils because $\mathrm{Cd}$ cover cuts off thermal-flux on the $\mathrm{Au}+\mathrm{Cd}$ foils, which measures radioactivities induced by neutron-flux of epi-thermal energy 
range (epi-thermal-flux: energy within $1.0 \mathrm{eV}$ to $1.0 \mathrm{MeV}$ ). We used Ni foils as threshold detectors to measure radioactivities induced by neutron-flux of fast energy range (fast-flux: energy higher than $1.0 \mathrm{MeV}$ ).

Activation foils at location 1 to 15 were irradiated from Feb. $17^{\text {th }} 2001$ to Feb. $25^{\text {th }} 2002 ; 373$ days. Further, we added fifteen locations at 16 to 30 from Oct. $26^{\text {th }} 2004$ to Nov. $11^{\text {th }} 2005 ; 381$ days.

Locations of activation foil in the PCV are shown in Figure 3. We set foils at thirty locations where characteristics of neutron transport phenomena in the PCV can be observed [4]. To observe neutron streaming caused by a Feed Water (FW) pipe, we set 6 sets of activation foils around the pipe as shown in the upper right corner in the figure.

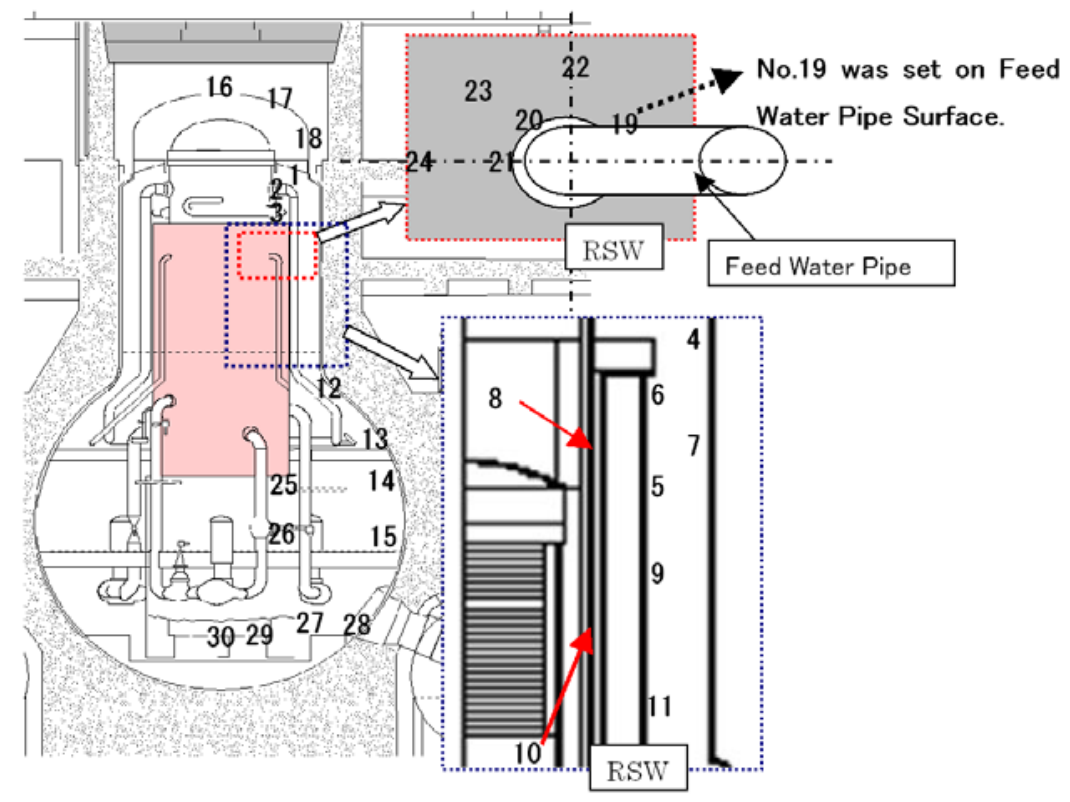

Figure 3 Locations of activation foil in the PCV of the BSW

\section{Activation calculations}

\subsection{Calculation conditions}

We performed activation calculations on irradiation conditions of each foil location by using SCALE5.1/ORIGEN-S [1]. We input spectrum indexes, which are input data of ORIGEN-S code, given by three group neutron-fluxes at each location, which were obtained from two dimensional (2D) distribution calculation of neutron-flux in our previous work [4]. Distribution of spectrum indexes such as RES, Fast and $\Phi_{\text {th }}$ are shown in Figure 4 (Here, $\Phi_{\text {th }}$ indicates distribution of thermal flux). We extracted values of the spectrum indexes at each foil location from the distributions to input ORIGEN-S code. We also input irradiation histories as mentioned above.

In the calculations of this work, we applied MAXS library collapsing with the spectrum of (7) in Figure 2. The spectrum of (7) is typical one in the PCV which shows a peak in epi-thermal energy range. 
Applying the library with the spectrum of (7) to the activation calculations in the PCV would be appropriate.

However, applying the library to the calculation of foil locations around the FW pipe might be inappropriate because effects of neutron-streaming around the FW pipe make distribution of neutron-flux around it complex.

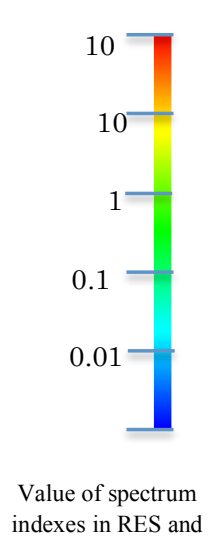

Fast

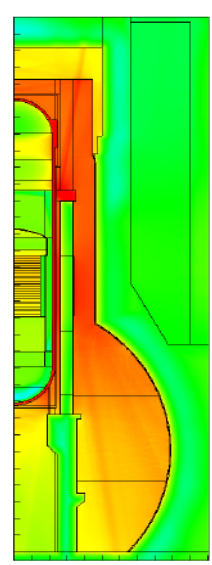

RES

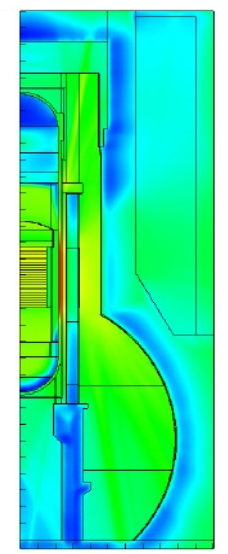

Fast

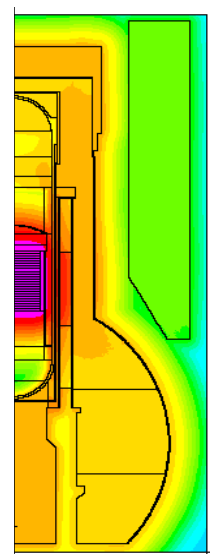

$\Phi_{t h}$

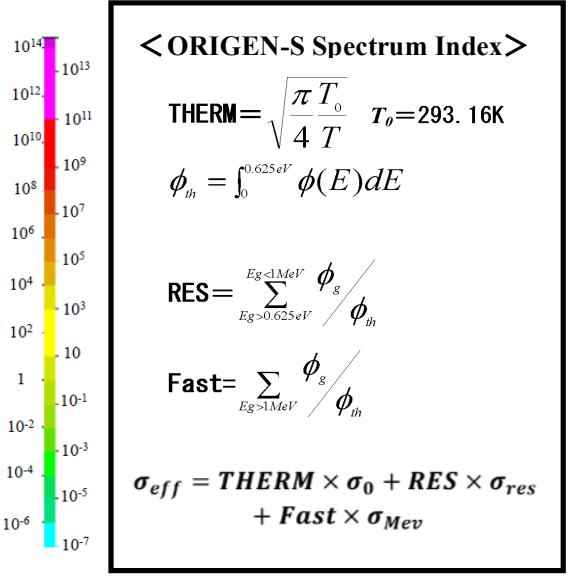

$\Phi$ th is distribution

of thermal-flux

$\left(\# /\left(\mathrm{cm}^{2} \mathrm{sec}\right)\right)$

Figure 4 Distribution of ORIGEN spectrum indexes

\subsection{Calculation results}

In works to be related to estimations of radioactivity for decommissioning, reliability of calculated radioactivity is verified with measured radioactivity. We judge calculation radioactivity distribution "Reliable", when comparison between calculated value and measured value $(\mathrm{C} / \mathrm{M})$ is less than one order magnitude $(1.0<\mathrm{C} / \mathrm{M}<10.0)[5] . \quad \mathrm{C} / \mathrm{Ms}$ of the calculations with ORIGEN library and with MAXS library at each foil location are shown in Figure 5. C/Ms of $\mathrm{Au}$ foils and $\mathrm{Ni}$ foils, which were calculated both with ORIGEN library and with MAXS library, are shown in the figure. When C/M nears 1.0, it means that calculated value simulates measured value well. C/Ms with MAXS library are smaller than those with ORIGEN library by almost half.

In $\mathrm{C} / \mathrm{Ms}$ of $\mathrm{Au}$ foils, MAXS library is effective to improve the reliability of the activation calculations in the PCV except locations around the PCV as seen in the figure. $\mathrm{C} / \mathrm{Ms}$ of these locations were less than 1.0. $\mathrm{C} / \mathrm{M}$, which is less than 1.0, indicates that calculation is underestimation. We suppose that the underestimation would result from the complex phenomena around FW pipe as mentioned above. 


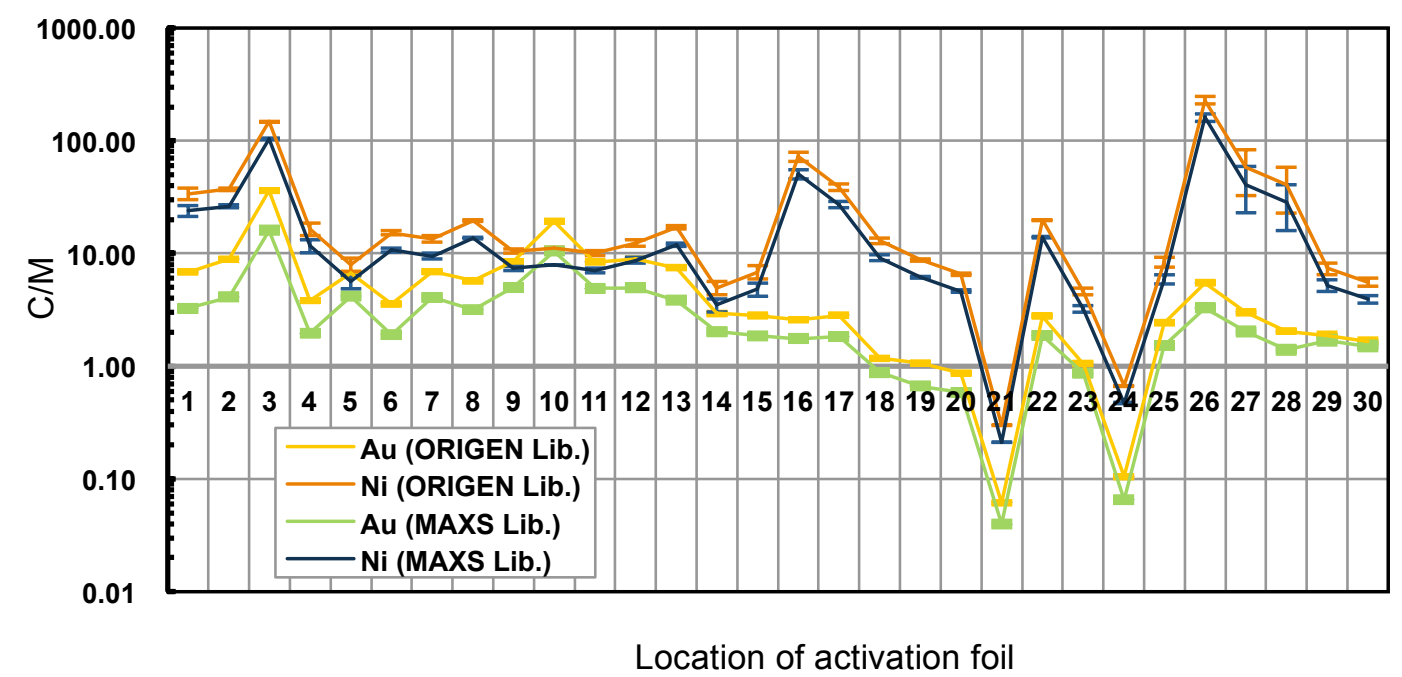

Figure 5 Comparisons between calculated and measured radioactiveities (C/M) with ORIGEN library and with

MAXS library at each foil location

In $\mathrm{C} / \mathrm{Ms}$ of $\mathrm{Ni}$ foils, almost all $\mathrm{C} / \mathrm{Ms}$ with ORIGEN library were larger than 10.0. These means calculations were overestimation. Although applying MAXS library improved C/Ms, Applying it was not so effective as those of $\mathrm{Au}$ foils. Activation reaction of $\mathrm{Ni}$ foil has threshold energy. ORIGEN-S code performs activation calculation with not fast neutron-flux but with total neutron-flux. Since using total neutron-flux as irradiation neutron would cause the overestimation, the effectiveness of the library could not made clear. C/Ms at foil locations around the FW pipe shows similar tendency as Au foils.

\section{Summary and conclusions}

We performed activation calculations at the locations in the PCV of the BWR where radioactiveities of both $\mathrm{Au}$ and Ni were measured as benchmark calculation for MAXS library. To examine an applicability of the library, we compered calculated radioactivities with measured ones. For Au foils, the calculations provided the applicability of applying MAXS library except around FW pipe where the complex phenomena of neutron transport would occur. We suppose that the library would be effective to the other nuclides of which activation reaction cross section spread through whole energy range that the activation calculations deal with. On the other hand, the applicability of the library for Ni foils were not so clear because of the threshold energy of activation reaction of $\mathrm{Ni}$.

In this work, only one spectrum was used to generate the library. The underestimations shown around FW pipe would be improved by applying libraries which were generated with appropriate spectra in the PCV. Activation calculations of nuclides, which have threshold energy of activation reaction, activation calculations might need not only libraries generated with appropriate spectra but also neutron-flux of irradiation. 


\section{References}

1. I.C.Gauld et.al, ORNL/TM - 2005/39 Version5.1 Vol.II, Book1, Sect.F7, (2006)

2. K. Okumura et.al, Proc. of SND 2014 $\square 150716$ (2014)

3. K. Tanaka et al, PNE,85: 254-270 (2015)

4. I.C.Gauld et.al, ORNL/TM - 2005/39 Version5.1 Vol.III, Sect.M6, (2006)

5. IAEA, Safety Series No.100, ISBN 9201240899 (1989) 